\title{
SNR G39.2-0.3, an hadronic cosmic rays accelerator
}

\author{
lurii Sushch, ${ }^{a, b, c, *}$ Emma de Oña Wilhelmi, ${ }^{c, d, e}$ Robert Brose, ${ }^{f, g}$ Enrique Mestre, ${ }^{d, e}$ \\ Yang $\mathrm{Su}^{h}$ and Roberta Zanin ${ }^{i}$ \\ ${ }^{a}$ Centre for Space Research, North-West University, 2520 Potchefstroom, South Africa \\ ${ }^{b}$ Astronomical Observatory of Ivan Franko National University of Lviv, Kyryla i Methodia 8, 79005 Lviv, \\ Ukraine \\ ${ }^{c}$ Deutsches Elektronen Synchrotron DESY, 15738 Zeuthen, Germany \\ ${ }^{d}$ Institute of Space Sciences (ICE/CSIC), Campus UAB, Carrer de Can Magrans s/n, 08193 Barcelona, \\ Spain \\ e Institut d'Estudis Espacials de Catalunya (IEEC), 08034 Barcelona, Spain \\ ${ }^{f}$ Dublin Institute for Advanced Studies, Astronomy \& Astrophysics Section, 31 Fitzwilliam Place, D02 \\ XF86 Dublin 2, Ireland \\ ${ }^{g}$ Institute of Physics and Astronomy, University of Potsdam, 14476 Potsdam, Germany \\ ${ }^{h}$ Purple Mountain Observatory and Key Laboratory of Radio Astronomy, Chinese Academy of Sciences, \\ Nanjing 210034, China \\ ${ }^{i}$ CTA Observatory GmbH, Via Piero Gobetti 93, I-40129 Bologna, Italy \\ E-mail: iurii.sushch@nwu.ac.za, emma.de.ona.wilhelmi@desy.de
}

\footnotetext{
${ }^{*}$ Presenter
} 
Recent results obtained with gamma-ray satellites have established supernova remnants as accelerators of $\mathrm{GeV}$ hadronic cosmic rays, which produce detectable gamma-ray emission through interaction with particles from gas clouds in their surrounding. In particular, the rich medium in which core-collapse SNRs explode provides a large target density to boost hadronic gamma-rays. SNR G39.2-0.3 is one of the brightest SNRs in infrared wavelengths, and its broad multiwavelength coverage allows a detailed modelling of its radiation from radio to high energies. The collection of the available multiwavelength data certainly favours a hadronic origin of the gamma-ray emission. The GeV spectrum obtained from the re-analysis of the Fermi LAT data implies that the present acceleration of protons is limited to below $10 \mathrm{GeV}$, which in turn points to a drastic slow down of the shock velocity due to the dense wall traced by the $\mathrm{CO}$ observations, surrounding the remnant. Further investigation of the gamma-ray spectral shape points to a dynamically old remnant subjected to severe escape of CRs and a decrease of acceleration efficiency. The low-energy (below 1 $\mathrm{GeV}$ ) peak of the gamma-ray spectrum, which suggests extremely low maximum energy of freshly accelerated protons, is, however, not easily reproduced within typical acceleration and evolution models. We discuss several scenarios that may explain such a spectral shape, one of which is the heavy composition of accelerated particles which is certainly expected for a core-collapse SNR with an environment enriched by heavy nuclei from stellar winds of the progenitor star. We also show that a widely discussed idea of gamma-ray production by compressed and re-accelerated pre-existing Galactic cosmic rays cannot explain the observed emission.

$37^{\text {th }}$ International Cosmic Ray Conference (ICRC 2021)

July 12th-23rd, 2021

Online - Berlin, Germany 
Results of this study were published as an article in Monthly Notices of the Royal Astronomical Society, Volume 497, Issue 3, September 2020, Pages 3581-3590. The article can be found at this link. 